\title{
LEXIFYING IDEALS
}

\author{
Jeffrey Mermin and Irena Peeva
}

\section{Introduction}

Let $B=k\left[x_{1}, \ldots, x_{n}\right]$ be a polynomial ring over a field $k$ graded by $\operatorname{deg}\left(x_{i}\right)=1$ for all $i$. What are the possible Hilbert functions of graded ideals in $B$ ? This question was answered by Macaulay [Ma], who showed that for every graded ideal there exists a lexicographic ideal with the same Hilbert function. Lexicographic ideals are highly structured: they are defined combinatorially and it is easy to derive the inequalities characterizing their possible Hilbert functions. Macaulay's Theorem also plays an important role in the study of graded $B$-ideals; for example,

- Hartshorne's [Ha] proof that the Hilbert scheme is connected uses lexicographic ideals in an essential way.

- The homological properties of lexicographic ideals are combinatorially tractable [EK]. This leads to results by Bigatti [Bi], Hulett [Hu], Pardue [Pa], showing that the lexicographic ideals have extremal Betti numbers.

Let $M$ be a monomial ideal. We say that a graded ideal in $B / M$ is lexifiable if there exists a lexicographic ideal in $B / M$ with the same Hilbert function. We call $M$ and $B / M$ Macaulay-Lex if every graded ideal in $B / M$ is lexifiable. The following results are well known: Macaulay's Theorem [Ma] says that 0 is a Macaulay-Lex ideal, Kruskal-Katona's Theorem [Ka, Kr] says that $\left(x_{1}^{2}, \ldots, x_{n}^{2}\right)$ is a Macaulay-Lex ideal, and Clements-Lindström's Theorem [CL] says that $\left(x_{1}^{e_{1}}, \ldots, x_{n}^{e_{n}}\right)$ is a MacaulayLex ideal if $e_{1} \leq \cdots \leq e_{n} \leq \infty$. These theorems are well-known and have many applications in Commutative Algebra, Combinatorics, and Algebraic Geometry.

It is easy to construct examples like Example 2.13, where a given Hilbert function is not attained by any lexicographic ideal in the degrees of the minimal generators of $M$. This motivated us to slightly weaken the definition: Let $q$ be the maximal degree of a minimal monomial generator of $M$; we call $M$ and $B / M$ pro-lex if every graded ideal generated in degrees $\geq q$ in $B / M$ is lexifiable. There exist examples of non pro-lex rings; see Example 3.15. We have started working in a new direction of research along the lines of the following problem.

Problem 1.1. Find classes of pro-lex monomial ideals.

Theorem 5.1 shows that if $M$ is Macaulay-Lex and $N$ is lexicographic, then $M+N$ is Macaulay-Lex. Theorem 4.1 shows that if $M$ is Macaulay-Lex, then it stays MacaulayLex after we add extra variables to the ring $B$. In Section 3 we prove:

Received by the editors October 24, 2004.

2000 Mathematics Subject Classification: 13F20.

Keywords and Phrases:Hilbert function, lexicographic ideals. 
Theorem 1.2. Let $P=\left(x_{1}^{e_{1}}, \cdots, x_{n}^{e_{n}}\right)$, with $e_{1} \leq e_{2} \leq \cdots \leq e_{n} \leq \infty\left(\right.$ here $\left.x_{i}^{\infty}=0\right)$, and $M$ be a compressed monomial ideal in $B / P$ generated in degrees $\leq p$. If $n=2$, assume that $M$ is $(B / P)$-lex. Set $\Upsilon=B /(M+P)$. Then $\Upsilon$ is pro-lex above $p$, that is, for every graded ideal $\Gamma$ in $\Upsilon$ generated in degrees $\geq p$ there exists an $\Upsilon$-lex ideal $\Theta$ with the same Hilbert function.

In the case when $M=P=0$, Theorem 1.2 is Macaulay's Theorem [Ma]; in the case when $M=0$, Theorem 1.2 is Clements-Lindström's Theorem [CL]. Examples 3.14 and 3.15 show that there are obstructions to generalizing Theorem 1.2.

We make use of ideas of Bigatti [Bi], Clements and Lindström [CL], and Green [Gr]. Our proofs are algebraic, and we avoid computations using generic forms (used in [Gr]) and combinatorial counting (used in [CL]). In Section 2 we introduce definitions and notation used throughout the paper.

\section{Lexification}

The notation in this section will be used throughout the paper. We introduce several definitions.

Let $k$ be a field and $B=k\left[x_{1}, \ldots, x_{n}\right]$ be graded by $\operatorname{deg}\left(x_{i}\right)=1$ for all $i$. We denote by $B_{d}$ the $k$-vector space spanned by all monomials of degree $d$. Denote $\mathbf{m}=\left(x_{1}, \ldots, x_{n}\right)_{1}$ the $k$-vector space spanned by the variables. We order the variables $x_{1}>\cdots>x_{n}$, and denote by $\succ_{\text {lex }}$ the homogeneous lexicographic order on the monomials. We say that an ideal is p-generated if it has a system of generators of degree $p$.

A monomial $x_{1}^{a_{1}} \ldots x_{n}^{a_{n}}$ has exponent vector $\mathbf{a}=\left(a_{1}, \ldots, a_{n}\right)$ and is denoted by $\mathbf{x}^{\mathbf{a}}$. For a monomial $m$, we set $\max (m)=\max \left\{i \mid x_{i}\right.$ divides $\left.m\right\}$. An ideal is called monomial if it can be generated by monomials; such an ideal has a unique minimal system of monomial generators.

Notation 2.1. Let $M$ be a monomial ideal. Set $\Upsilon=B / M$. Vector spaces in $\Upsilon$ (and sometimes ideals) are denoted by greek letters. For example, we denote by $C_{d}$ a subspace of $B_{d}$, and we denote by $\tau_{d}$ a subspace of $\Upsilon_{d}$.

Definition 2.2. A monomial is a product of powers of the variables, so it can be considered as an element in either $B$ or $\Upsilon$. We say that a monomial is an $\Upsilon$-monomial if it does not vanish in $\Upsilon$, that is, it is not in $M$. We say that a monomial is an $\Upsilon_{d}$-monomial if it is an $\Upsilon$-monomial of degree $d$. Furthermore, we say that $\tau_{d}$ is an $\Upsilon_{d}$-monomial space if it can be spanned by $\Upsilon_{d}$-monomials. We denote by $\left\{\tau_{d}\right\}$ the set of $\Upsilon_{d}$-monomials contained in $\tau_{d}$. The cardinality of this set is $\left|\tau_{d}\right|=\operatorname{dim}_{k} \tau_{d}$. By $\mathbf{m} \tau_{d}$ we mean the $k$-vector subspace $\left(\mathbf{m}\left(\tau_{d}\right)\right)_{d+1}$ of $\Upsilon_{d+1}$.

Definition 2.3. Let $L$ be a monomial ideal in $\Upsilon$ minimally generated by $\Upsilon$-monomials $l_{1}, \ldots, l_{r}$. We say that $L$ is $\Upsilon$-lex, ( $\Upsilon$-lexicographic), if the following property is satisfied:

$$
\left.\begin{array}{c}
m \text { is an } \Upsilon \text {-monomial } \\
m \succ_{\text {lex }} l_{i} \text { and } \operatorname{deg}(m)=\operatorname{deg}\left(l_{i}\right) \text {, for some } 1 \leq i \leq r
\end{array}\right\} \quad \Longrightarrow \quad m \in L .
$$

The $\Upsilon_{d}$-lex-segment $\lambda_{d, p}$ of length $p$ in degree $d$ is defined as the $k$-vector space spanned by the lexicographically first (greatest) $p$ monomials in $\Upsilon_{d}$. We say that $\lambda_{d}$ 
is a lex-segment in $\Upsilon_{d}$ if there exists a $p$ such that $\lambda_{d}=\lambda_{d, p}$. For a $\Upsilon_{d}$-monomial space $\tau_{d}$, we say that $\lambda_{d,\left|\tau_{d}\right|}$ is its $\Upsilon_{d}$-lexification.

For simplicity, we sometimes say lex instead of $\Upsilon$-lex if it is clear over which ring we work.

Example 2.4. The ideal $\left(a^{2}, a b, b^{2}\right)$ is lex in the ring $k[a, b, c, d] /(a c, a d)$, and its generators span a lex-segment. The $k$-vector space spanned by $a^{2}, a b, b^{2}$ is the lexification of the k-vector space spanned by $b^{2}, c^{2}, c d$. However, the ideal is not lex in $k[a, b, c, d]$.

Proposition 2.5. If $\tau_{d}$ is an $\Upsilon_{d}$-lex-segment, then $\mathbf{m} \tau_{d}$ is an $\Upsilon_{d+1}$-lex-segment.

The proofs of Propositions 2.5, 2.7, and 2.8, are easy and very similar to the proofs in the polynomial case.

Definition 2.6. A monomial $m^{\prime}$ is said to be in the big shadow of a monomial $m$ if $m^{\prime}=\frac{x_{i} m}{x_{j}}$ for some $x_{j}$ dividing $m$ and some $i \leq j$. A monomial ideal in $\Upsilon$ is $\Upsilon$-Borel if it contains all $\Upsilon$-monomials in the big shadows of its minimal $\Upsilon$-monomial generators. Ideals that are B-Borel are usually called strongly stable or 0-Borel fixed. We say that a monomial space $\tau_{d}$ is $\Upsilon_{d}$-Borel if it contains all $\Upsilon_{d}$-monomials in the big shadows of its monomial generators.

Proposition 2.7. If $\tau_{d}$ is $\Upsilon_{d}$-Borel, then $\mathbf{m} \tau_{d}$ is $\Upsilon_{d+1}$-Borel.

Proposition 2.8. If $\tau_{d}$ is an $\Upsilon_{d}$-lex-segment, then it is $\Upsilon_{d}$-Borel.

Notation 2.9. Let $\Gamma$ be a graded ideal in $\Upsilon$. It decomposes as a direct sum of its components $\Gamma=\oplus_{d \geq 0} \Gamma_{d}$. Its Hilbert function $\operatorname{Hilb}_{\Gamma}^{\Upsilon}: \mathbf{N} \cup 0 \rightarrow \mathbf{N} \cup 0$ is defined by

$$
\operatorname{Hilb}_{\Gamma}^{\Upsilon}(d)=\operatorname{dim}_{k}\left(\Gamma_{d}\right) \quad \text { for all } d \geq 0 .
$$

We use the notation $\left|\Gamma_{d}\right|^{\Upsilon}=\operatorname{Hilb}_{\Gamma}^{\Upsilon}(d)$; and for simplicity, we write $\left|\Gamma_{d}\right|$ if it is clear over which ring we work.

Definition 2.10. We say that a graded ideal $R$ in $\Upsilon$ is lexifiable if there exists an $\Upsilon$ lex ideal with the same Hilbert function as $R$. The monomial ideal $M$ and the quotient ring $\Upsilon=B / M$ are called Macaulay-Lex if every graded ideal in $\Upsilon$ is lexifiable.

Given an $\Upsilon_{d}$-monomial space $\tau_{d}$, the number $\left|\mathbf{m} \tau_{d}\right|$ measures the dimension of $\left(\tau_{d}\right)_{d+1}$. In the proofs one usually works in a fixed degree, so we need the next variation of Definition 2.10.

Definition 2.11. We say that an $\Upsilon_{d}$-monomial space $\tau_{d}$ is $\Upsilon_{d}$-lexifiable if its lexification $\lambda_{d}$ has the property that $\left|\mathbf{m} \lambda_{d}\right| \leq\left|\mathbf{m} \tau_{d}\right|$. The monomial ideal $M$ and the quotient ring $\Upsilon=B / M$ are called d-pro-lex, if every $\Upsilon_{d}$-monomial space is $\Upsilon_{d}$-lexifiable.

Example 2.12. This example shows that the order of the variables can make a difference. The ideal $(a b)$ is not lexifiable in the ring $k[a, b] /\left(a b^{2}\right)$ for the lex order with $a>b$, but it is lexifiable for the lex order with $b>a$.

Example 2.13. The ideal $(a b)$ is not lexifiable in the ring $k[a, b] /\left(a^{2} b, a b^{2}\right)$ in any lex order. 
It is easy to construct many examples like Example 2.13. This observation suggests that in order to obtain positive results we need to slightly relax Definition 2.10; the following definition seems reasonable to us:

Definition 2.14. Let $q$ be the maximal degree of a minimal monomial generator of $M$. The monomial ideal $M$ and the quotient ring $\Upsilon=B / M$ are called pro-lex if every graded ideal generated in degrees $\geq q$ in $\Upsilon$ is lexifiable.

In the examples we usually denote the variables by $a, b, c, d$ for simplicity.

\section{Compression}

The following definition generalizes a definition introduced by Clements and Lindström [CL], who used it over a quotient of a polynomial ring modulo pure powers of the variables.

Definition 3.1. Let $E$ be a monomial ideal in $B . A(B / E)_{d}$-monomial space $\tau_{d}$ is called $i$-compressed (or $i$-compressed in $\left.(B / E)_{d}\right)$ if we have the disjoint union

$$
\left\{\tau_{d}\right\}=\coprod_{0 \leq j \leq d} x_{i}^{d-j}\left\{\sigma_{j}\right\}
$$

and each $\sigma_{j}$ is a lex-segment in $\left(B /\left(E, x_{i}\right)\right)_{j}$. We say that a k-vector space $\tau_{d}$ is $(B / E)_{d}$-compressed (or compressed) if it is a $(B / E)_{d}$-monomial space and is $i$ compressed for all $1 \leq i \leq n$. A monomial ideal $W$ in $B / E$ is called compressed if $W_{d}$ is compressed for all $d \geq 0$. The term "compressed" is used by Clements and Lindström [CL].

Example 3.2. The ideal

$$
\left(a^{3}, a^{2} b, a^{2} c, a b^{2}, a b c, b^{3}, b^{2} c\right)
$$

is compressed in the ring $k[a, b, c]$. The ideal

$$
M=\left(a^{3}, a^{2} b, a^{2} c, a^{2} d, a b^{2}, a b c, a b d, b^{3}, b^{2} c\right)
$$

is Borel, but not compressed (it is not c-compressed because $b^{3} \in M$ and $a d^{2} \notin M$ ).

Lemma 3.3. If $\tau_{d}$ is $i$-compressed in $(B / E)_{d}$ then $\mathbf{m} \tau_{d}$ is $i$-compressed in $(B / E)_{d+1}$. If $\tau_{d}$ is $\left.(B / E)\right)_{d}$-lex, then it is $(B / E)_{d}$-compressed.

Definition 3.4. A B-monomial ideal $K$ is called compressed-plus-powers if $K=$ $M+P$, where $P=\left(x_{1}^{e_{1}}, \cdots, x_{n}^{e_{n}}\right)$ with $e_{1} \leq e_{2} \leq \cdots \leq e_{n} \leq \infty$ and the monomial ideal $M$ is compressed in $B / P$. Sometimes, when we need to be more precise, we say that $K$ is compressed-plus-P. Furthermore, we say that $K$ is lex-plus- $P$ if $M$ is lex in $B / P$.

Notation 3.5. Throughout this section we use the following notation and assumptions:

- $P=\left(x_{1}^{e_{1}}, \cdots, x_{n}^{e_{n}}\right)$ with $2 \leq e_{1} \leq e_{2} \leq \cdots \leq e_{n} \leq \infty$.

- The ideal $K=M+P$ is a compressed-plus-P monomial ideal in $B$; here $M$ is compressed in $B / P$.

- If $n=2$ we assume in addition that $K$ is lex-plus-P. 
- Except in Theorem 3.12, we assume that $M$ is generated by monomials in degree $p$.

- Set $\Upsilon=B / K$.

For a $(B / P)_{d}$-monomial space $A_{d}$ set

$$
\begin{aligned}
& t_{i}\left(A_{d}\right)=\left|\left\{m \in\left\{A_{d}\right\} \mid \max (m) \leq i\right\}\right| \\
& s_{i}\left(A_{d}\right)=\mid\left\{m \in\left\{A_{d}\right\} \mid \max (m)=i \text { and } x_{i}^{e_{i}-1} \text { divides } m\right\} \mid \\
& r_{i, j}\left(A_{d}\right)=\mid\left\{m \in\left\{A_{d}\right\} \mid \max (m) \leq i \text { and } x_{i}^{j} \text { does not divide } m\right\} \mid .
\end{aligned}
$$

The formula in the following lemma is a generalization of a formula introduced by Bigatti [Bi], who used it for $B$-Borel ideals.

Lemma 3.6. Let $A_{d}$ be a $(B / P)_{d}$-monomial space.

1. If $A_{d}$ is compressed and $n \geq 3$, then $A_{d}$ is $(B / P)_{d}$-Borel.

2. If $A_{d}$ is $(B / P)_{d}$-Borel, then

$$
\left|\left\{\mathbf{m} A_{d}\right\}\right|=\sum_{i=1}^{n} t_{i}\left(A_{d}\right)-s_{i}\left(A_{d}\right)=\sum_{i=1}^{n} r_{i, e_{i}-1}\left(A_{d}\right) .
$$

Proof. First, we prove (1). Let $m \in\left\{A_{d}\right\}$ and $m^{\prime}$ be a $(B / P)_{d}-$ monomial in its big shadow. Hence $m^{\prime}=\frac{x_{i} m}{x_{j}}$ for some $x_{j}$ dividing $m$ and some $i \leq j$. There exists an index $1 \leq q \leq n$ such that $q \neq i, j$. Note that that $m$ and $m^{\prime}$ have the same $q$-exponents. Since $A_{d}$ is $q$-compressed and $m^{\prime} \succ_{\text {lex }} m$, it follows that $m^{\prime} \in\left\{A_{d}\right\}$. Therefore, $A_{d}$ is $(B / P)_{d}$-Borel.

Now, we prove (2). We will show that $\left\{\mathbf{m} A_{d}\right\}$ is equal to the set

$$
\begin{aligned}
& \coprod_{i=1}^{n} x_{i}\left\{m \in\left\{A_{d}\right\} \mid \max (m) \leq i\right\} \\
& \backslash \coprod_{i=1}^{n} x_{i}\left\{m \in\left\{A_{d}\right\} \mid \max (m)=i \text { and } x_{i}^{e_{i}-1} \text { divides } m\right\} .
\end{aligned}
$$

Denote by $\mathcal{P}$ the set above. Let $w \in A_{d}$. For $j \geq \max (w)$ we have that $x_{j} w \in \mathcal{P}$. Let $j<\max (w)$. Then $v=x_{j} \frac{w}{x_{\max (w)}} \in A_{d}$. So, $x_{j} w=x_{\max (w)} v \in \mathcal{P}$.

Lemma 3.7 is a generalization of a result by M. Green [Gr], who proved a particular case of it it over a polynomial ring (in the case $M=0$ ). Green's proof is entirely different than ours; he makes a computation with generic linear forms. It is not clear how to apply his computation to the case $M \neq 0$.

Lemma 3.7. Let $\tau_{d}$ be an n-compressed Borel $\Upsilon_{d}$-monomial space, and let $\lambda_{d}$ be a lex-segment in $\Upsilon_{d}$ with $\left|\left\{\lambda_{d}\right\}\right| \leq\left|\left\{\tau_{d}\right\}\right|$. Let $L_{d}$ and $T_{d}$ be the $(B / P)_{d}$-monomial spaces such that $\left\{L_{d}\right\}=\left\{\lambda_{d}\right\} \coprod\left\{M_{d}\right\}$ and $\left\{T_{d}\right\}=\left\{\tau_{d}\right\} \coprod\left\{M_{d}\right\}$. For each $1 \leq i \leq n$ and each $1 \leq j \leq e_{i}$ we have

$$
r_{i, j}\left(L_{d}\right) \leq r_{i, j}\left(T_{d}\right)
$$


Proof. Set $R=B / P$. By Lemma 3.6, $M_{d}$ is $R_{d}$-Borel. Therefore, both $L_{d}$ and $T_{d}$ are $R_{d}$-Borel and $n$-compressed.

First, we consider the case $i=n$. Clearly, $r_{n, e_{n}}\left(L_{d}\right)=\left|L_{d}\right|=\left|T_{d}\right|=r_{n, e_{n}}\left(T_{d}\right)$ (if $e_{n}=\infty$, then we consider $r_{n, d+1}$ here). We induct on $j$ decreasingly. Suppose that $r_{i, j+1}\left(L_{d}\right) \leq r_{i, j+1}\left(T_{d}\right)$ holds by induction.

If $\left\{T_{d}\right\}$ contains no monomial divisible by $x_{n}^{j}$ then

$$
r_{i, j}\left(L_{d}\right) \leq r_{i, j+1}\left(L_{d}\right) \leq r_{i, j+1}\left(T_{d}\right)=r_{i, j}\left(T_{d}\right) .
$$

Suppose that $\left\{T_{d}\right\}$ contains a monomial divisible by $x_{n}^{j}$. Denote by $e=x_{1}^{b_{1}} \ldots x_{n}^{b_{n}}$, with $b_{n} \geq j$, the lex-smallest monomial in $T_{d}$ that is divisible by $x_{n}^{j}$. Let $0 \leq q \leq j-1$. Since $T_{d}$ is $R_{d}$-Borel, it follows that $c_{q}=x_{n-1}^{b_{n}-q} \frac{e}{x_{n}^{b_{n}-q}} \in T_{d}$. This is the lex-smallest monomial that is lex-greater than $e$ and $x_{n}$ divides it at power $q$. Let the monomial $a=x_{1}^{a_{1}} \ldots x_{n-1}^{a_{n-1}} x_{n}^{q} \in R_{d}$ be lex-greater than $e$. Since $T_{d}$ is $n$-compressed and $a$ is lex-greater (or equal) than $c_{q}$, it follows that $a \in T_{d}$.

For a monomial $u$, we denote by $x_{n} \notin u$ the property that $x_{n}^{j}$ does not divide $u$. By what we proved above, it follows that

$$
\left|\left\{u \in\left\{T_{d}\right\} \mid x_{n} \notin u, u \succ_{\text {lex }} e\right\}\right|=\left|\left\{u \in\left\{R_{d}\right\} \mid x_{n} \notin u, u \succ_{\text {lex }} e\right\}\right| .
$$

Therefore,

$$
\begin{aligned}
r_{i, j}\left(L_{d}\right) & =\left|\left\{u \in\left\{L_{d}\right\} \mid x_{n} \notin u, u \succ_{\text {lex }} e\right\}\right|+\left|\left\{u \in\left\{L_{d}\right\} \mid x_{n} \notin u, u \prec_{\text {lex }} e\right\}\right| \\
& \leq\left|\left\{u \in\left\{R_{d}\right\} \mid x_{n} \notin u, u \succ_{\text {lex }} e\right\}\right|+\left|\left\{u \in\left\{L_{d}\right\} \mid x_{n} \notin u, u \prec_{\text {lex }} e\right\}\right| \\
& \leq\left|\left\{u \in\left\{R_{d}\right\} \mid x_{n} \notin u, u \succ_{\text {lex }} e\right\}\right|+\left|\left\{u \in\left\{L_{d}\right\} \mid u \prec_{\text {lex }} e\right\}\right| \\
& \leq\left|\left\{u \in\left\{R_{d}\right\} \mid x_{n} \notin u, u \succ_{\text {lex }} e\right\}\right|+\left|\left\{u \in\left\{T_{d}\right\} \mid u \prec_{\text {lex }} e\right\}\right| \\
& =\left|\left\{u \in\left\{R_{d}\right\} \mid x_{n} \notin u, u \succ_{\text {lex }} e\right\}\right|+\left|\left\{u \in\left\{T_{d}\right\} \mid x_{n} \notin u, u \prec_{\text {lex }} e\right\}\right| \\
& =\left|\left\{u \in\left\{T_{d}\right\} \mid x_{n} \notin u, u \succ_{\text {lex }} e\right\}\right|+\left|\left\{u \in\left\{T_{d}\right\} \mid x_{n} \notin u, u \prec_{\text {lex }} e\right\}\right| \\
& =r_{i, j}\left(T_{d}\right)
\end{aligned}
$$

for the third inequality we used the fact that $\lambda_{d}$ is a lex-segment in $\Upsilon_{d}$ with $\left|\left\{\lambda_{d}\right\}\right| \leq\left|\left\{\tau_{d}\right\}\right|$; for the equality after that we used the definition of $e$; for the next equality we used (3.8). Thus, we have the desired inequality in the case $i=n$.

In particular, we have proved that

$$
t_{n-1}\left(L_{d}\right)=r_{n, 1}\left(L_{d}\right) \leq r_{n, 1}\left(T_{d}\right)=t_{n-1}\left(T_{d}\right) .
$$

Finally, we prove the lemma for all $i<n$. Both $\left\{\tau_{d} / x_{n}\right\}$ and $\left\{\lambda_{d} / x_{n}\right\}$ are lexsegments in $\Upsilon_{d} / x_{n}$ since $\tau_{d}$ is $n$-compressed. By (3.9) the inequality $t_{n-1}\left(L_{d}\right) \leq$ $t_{n-1}\left(T_{d}\right)$ holds, and it implies the inclusion $\left\{\tau_{d} / x_{n}\right\} \supseteq\left\{\lambda_{d} / x_{n}\right\}$. The desired inequalities follow since

$$
\begin{aligned}
r_{i, j}\left(T_{d}\right) & =r_{i, j}\left(T_{d} /\left(x_{i+1}, \ldots, x_{n}\right)\right) \\
& =r_{i, j}\left(\left\{\tau_{d} /\left(x_{i+1}, \ldots, x_{n}\right)\right\} \coprod\left\{M_{d} /\left(x_{i+1}, \ldots, x_{n}\right)\right\}\right) \\
r_{i, j}\left(L_{d}\right) & =r_{i, j}\left(L_{d} /\left(x_{i+1}, \ldots, x_{n}\right)\right) \\
& =r_{i, j}\left(\left\{\lambda_{d} /\left(x_{i+1}, \ldots, x_{n}\right)\right\} \coprod\left\{M_{d} /\left(x_{i+1}, \ldots, x_{n}\right)\right\}\right) .
\end{aligned}
$$

Lemma 3.10. Let $v_{d}$ be a $\Upsilon_{d}$-monomial space. There exists a compressed monomial space $\tau_{d}$ in $\Upsilon_{d}$ such that $\left|\tau_{d}\right|=\left|v_{d}\right|$ and $\left|\mathbf{m} \tau_{d}\right| \leq\left|\mathbf{m} v_{d}\right|$. 
Proof. Suppose that $v_{d}$ is not $i$-compressed. Set $z=x_{i}$. Since $M$ is $z$-compressed in $B / P$, we have the disjoint union

$$
\left\{M_{d}\right\}=\coprod_{0 \leq j \leq d} z^{d-j}\left\{N_{j}\right\},
$$

where each $N_{j}$ is a $(B /(z, P))_{j}$-lex-segment.

We also have the disjoint union

$$
\left\{v_{d}\right\}=\coprod_{0 \leq j \leq d} z^{d-j}\left\{\nu_{j}\right\}
$$

where each $\nu_{j}$ is a monomial space in $B /\left(z, P, N_{j}\right)$. Let $\gamma_{j}$ be the lexification of the space $\nu_{j}$ in $B /\left(z, P, N_{j}\right)$. Consider the $\Upsilon_{d}$-monomial space $\tau_{d}$ defined by

$$
\left\{\tau_{d}\right\}=\coprod_{0 \leq j \leq d} z^{d-j}\left\{\gamma_{j}\right\}
$$

Clearly, $\left|\tau_{d}\right|=\left|v_{d}\right|$.

Consider the $(B / P)_{d}$-monomial spaces $V_{d}$ and $T_{d}$ such that

$$
\left\{V_{d}\right\}=\left\{v_{d}\right\} \coprod\left\{M_{d}\right\} \quad \text { and } \quad\left\{T_{d}\right\}=\left\{\tau_{d}\right\} \coprod\left\{M_{d}\right\}
$$

Set $R=B / P$. The short exact sequence of $k$-vector subspaces of $(B / P)_{d+1}$

$$
0 \rightarrow \mathbf{m} M_{d} \rightarrow \mathbf{m} T_{d} \longrightarrow \mathbf{m} T_{d} / \mathbf{m} M_{d}=\mathbf{m} \tau_{d} /\left(\mathbf{m} \tau_{d} \cap \mathbf{m} M_{d}\right) \rightarrow 0
$$

shows that $\left|\mathbf{m} \tau_{d}\right|=\left|\mathbf{m} T_{d}\right|-\left|\mathbf{m} M_{d}\right|$ (here we mean $\left|\mathbf{m} \tau_{d}\right|^{\Upsilon}=\left|\mathbf{m} T_{d}\right|^{B / P}-\left|\mathbf{m} M_{d}\right|^{B / P}$ ). Similarly, the short exact sequence of $k$-vector subspaces of $(B / P)_{d+1}$

$$
0 \rightarrow \mathbf{m} M_{d} \rightarrow \mathbf{m} V_{d} \longrightarrow \mathbf{m} V_{d} / \mathbf{m} M_{d}=\mathbf{m} v_{d} /\left(\mathbf{m} v_{d} \cap \mathbf{m} M_{d}\right) \rightarrow 0
$$

shows that $\left|\mathbf{m} v_{d}\right|=\left|\mathbf{m} V_{d}\right|-\left|\mathbf{m} M_{d}\right|$. Therefore, the desired inequality $\left|\mathbf{m} \tau_{d}\right| \leq\left|\mathbf{m} v_{d}\right|$ is equivalent to the inequality

$$
\left|\mathbf{m} T_{d}\right| \leq\left|\mathbf{m} V_{d}\right|
$$

We will prove the latter inequality.

We have the disjoint unions

$$
\begin{aligned}
& \left\{V_{d}\right\}=\coprod_{0 \leq j \leq d} z^{d-j}\left\{U_{j}\right\} \quad \text { and } \quad\left\{T_{d}\right\}=\coprod_{0 \leq j \leq d} z^{d-j}\left\{F_{j}\right\}, \quad \text { where } \\
& \left\{U_{j}\right\}=\left\{\nu_{j}\right\} \coprod\left\{N_{j}\right\} \quad \text { and } \quad\left\{F_{j}\right\}=\left\{\gamma_{j}\right\} \coprod\left\{N_{j}\right\} \quad \text { in the ring } B /(z, P) .
\end{aligned}
$$

Note that each $F_{j}$ is a $(B /(z, P))_{j}$-lex-segment. Furthermore, we have the disjoint unions

$$
\begin{aligned}
& \left\{\mathbf{m} V_{d}\right\}=\coprod_{0 \leq j \leq d} z^{d-j+1}\left\{U_{j}+\mathbf{n} U_{j-1}\right\} \\
& \left\{\mathbf{m} T_{d}\right\}=\coprod_{0 \leq j \leq d} z^{d-j+1}\left\{F_{j}+\mathbf{n} F_{j-1}\right\}
\end{aligned}
$$

where $\mathbf{n}=\mathbf{m} / z$. We will show that

$$
\left|F_{j}+\mathbf{n} F_{j-1}\right|=\max \left\{\left|F_{j}\right|,\left|\mathbf{n} F_{j-1}\right|\right\} \leq \max \left\{\left|U_{j}\right|,\left|\mathbf{n} U_{j-1}\right|\right\} \leq\left|U_{j}+\mathbf{n} U_{j-1}\right| .
$$


The first equality above holds because both $F_{j}$ and $\mathbf{n} F_{j-1}$ are $(B /(z, P))_{j}$-lex-segments, so $F_{j}+\mathbf{n} F_{j-1}$ is the longer of these two lex-segments. The last inequality is obvious. It remains to prove the middle inequality. Using the short exact sequences of $k$-vector subspaces of $(B / P)_{j}$

$$
\begin{aligned}
& 0 \rightarrow \mathbf{n} N_{j-1} \rightarrow \mathbf{n} F_{j-1} \longrightarrow \mathbf{n} F_{j-1} / \mathbf{n} N_{j-1}=\mathbf{n} \gamma_{j-1} /\left(\mathbf{n} \gamma_{j-1} \cap \mathbf{n} N_{j-1}\right) \rightarrow 0 \\
& 0 \rightarrow \mathbf{n} N_{j-1} \rightarrow \mathbf{n} U_{j-1} \longrightarrow \mathbf{n} U_{j-1} / \mathbf{n} N_{j-1}=\mathbf{n} \nu_{j-1} /\left(\mathbf{n} \nu_{j-1} \cap \mathbf{n} N_{j-1}\right) \rightarrow 0
\end{aligned}
$$

we get $\left|\mathbf{n} \gamma_{j-1}\right|=\left|\mathbf{n} F_{j-1}\right|-\left|\mathbf{n} N_{j-1}\right|$ and $\left|\mathbf{n} \nu_{j-1}\right|=\left|\mathbf{n} U_{j-1}\right|-\left|\mathbf{n} N_{j-1}\right|$. Therefore, the desired inequality $\left|\mathbf{n} F_{j-1}\right| \leq\left|\mathbf{n} U_{j-1}\right|$ is equivalent to the inequality $\left|\mathbf{n} \gamma_{j-1}\right| \leq$ $\left|\mathbf{n} \nu_{j-1}\right|$. The latter inequality holds since by construction $\gamma_{j-1}$ is the lexification of $\nu_{j-1}$, so $\left|\gamma_{j-1}\right|=\left|\nu_{j-1}\right|$ and by induction on the number of variables we can apply Theorem 3.11 to the ring $B /\left(z, P, N_{j}\right)$.

Thus, $\left|F_{j}+\mathbf{n} F_{j-1}\right| \leq\left|U_{j}+\mathbf{n} U_{j-1}\right|$. Multiplication by $z^{d-j+1}$ is injective if $d-j+1 \leq$ $e_{i}-1$ and is zero otherwise, therefore we conclude that

$$
\left|z^{d-j+1}\left(F_{j}+\mathbf{n} F_{j-1}\right)\right| \leq\left|z^{d-j+1}\left(U_{j}+\mathbf{n} U_{j-1}\right)\right| .
$$

This implies the desired inequality $\left|\mathbf{m} T_{d}\right| \leq\left|\mathbf{m} V_{d}\right|$.

Note that $\left\{\tau_{d}\right\}$ is greater lexicographically than $\left\{v_{d}\right\}$. If $\tau_{d}$ is not compressed, we can apply the argument above. After finitely many steps in this way, the process must terminate because at each step we construct a lex-greater monomial space. Thus, after finitely many steps, we reach a compressed monomial space.

Theorem 3.11. Let $v_{d}$ be a $\Upsilon_{d}$-monomial space and $\lambda_{d}$ be its lexification in $\Upsilon_{d}$. Then $\left|\mathbf{m} \lambda_{d}\right| \leq\left|\mathbf{m} v_{d}\right|$.

Proof. The theorem clearly holds if $n=1$. Suppose that $n=2$. An easy calculation shows that the theorem holds, provided we do not have $e_{2} \leq d+1<e_{1}$. By the assumption on the ordering of the exponents, this does not hold and we are fine.

Suppose that $n \geq 3$. First, we apply Lemma 3.10 to reduce to the compressed case. We obtain a compressed $\Upsilon_{d}$-monomial space $\tau_{d}$ such that $\left|\tau_{d}\right|=\left|v_{d}\right|$ and $\left|\mathbf{m} \tau_{d}\right| \leq$ $\left|\mathbf{m} v_{d}\right|$. Let $L_{d}$ and $T_{d}$ be the $(B / P)_{d}$-monomial spaces such that $\left\{L_{d}\right\}=\left\{\lambda_{d}\right\} \cup\left\{M_{d}\right\}$ and $\left\{T_{d}\right\}=\left\{\tau_{d}\right\} \cup\left\{M_{d}\right\}$, where the disjoint unions take place in $B / P$. Both $L_{d}$ and

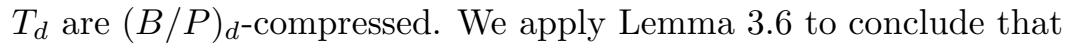

$$
\begin{aligned}
& \left|\left\{\mathbf{m} T_{d}\right\}\right|=\sum_{i=1}^{n} t_{i}\left(T_{d}\right)-\sum_{i=1}^{n} s_{i}\left(T_{d}\right) \\
& \left|\left\{\mathbf{m} L_{d}\right\}\right|=\sum_{i=1}^{n} t_{i}\left(L_{d}\right)-\sum_{i=1}^{n} s_{i}\left(L_{d}\right) .
\end{aligned}
$$

Finally, we apply Lemma 3.7 and conclude that $\left|\left\{\mathbf{m} L_{d}\right\}\right| \leq\left|\left\{\mathbf{m} T_{d}\right\}\right|$. This inequality and short exact sequences, as in the proof of Lemma 3.10, imply the desired $\left|\mathbf{m} \lambda_{d}\right| \leq$ $\left|\mathbf{m} v_{d}\right|$.

Equivalently, we obtain the following theorem, stated in the introduction:

Theorem 3.12. Let $P=\left(x_{1}^{e_{1}}, \cdots, x_{n}^{e_{n}}\right)$, with $e_{1} \leq e_{2} \leq \cdots \leq e_{n} \leq \infty$ (here $x_{i}^{\infty}=$ $0)$, and $M$ be a compressed monomial ideal in $B / P$ generated in degrees $\leq p$. If $n=2$, assume that $M$ is $(B / P)$-lex. Set $\Upsilon=B /(M+P)$. Then $\Upsilon$ is pro-lex above 
$p$, that is, for every graded ideal $\Gamma$ in $\Upsilon$ generated in degrees $\geq p$ there exists an $\Upsilon$-lex ideal $\Theta$ with the same Hilbert function.

Proof. We can assume that $\Gamma$ is a monomial ideal by Gröbner basis theory. For each $d \geq p$, let $\lambda_{d}$ be the lexification of $\Gamma_{d}$. By Theorem 3.11, it follows that $\Theta=\oplus_{d \geq p} \lambda_{d}$ is an ideal. By construction, it is a lex-ideal and has the same Hilbert function as $\Gamma$ in all degrees greater than or equal to $p$.

Remark 3.13. In the case when $M=P=0$, Theorem 3.12 is the well-known Macaulay's Theorem [Ma]. In the case $M=0$, Theorem 3.12 is the ClementsLindström's Theorem [CL].

Example 3.14. It is natural to ask if a compressed ideal is Macaulay-Lex. This example shows that the answer is negative. Take $P=0$. The ideal

$$
M=\left(a^{3}, a^{2} b, a^{2} c, a b^{2}, a b c, b^{3}, b^{2} c\right)
$$

is compressed (and Borel) in the ring $k[a, b, c]$. The ideal $\left(a^{2}, a b, b^{2}\right)$ in $k[a, b, c] / M$ is not lexifiable.

Example 3.15. It is natural to ask if Theorem 3.12 holds in the case when $M$ is a $B$-Borel ideal. It does not. Take $P=0$. The ideal

$$
M=\left(a^{3}, a^{2} b, a^{2} c, a^{2} d, a b^{2}, a b c, a b d, b^{3}, b^{2} c\right)
$$

is Borel in the ring $k[a, b, c, d]$. However it is not pro-lex because the ideal $\left(b^{2} d\right)$ is not lexifiable in $k[a, b, c] / M$.

\section{Adding new variables}

Theorem 4.1. If $B / M$ is Macaulay-Lex then $B[y] / M$ is Macaulay-Lex.

In this section, $W=B[y] / M, \mathbf{m}$ is the $k$-vector space spanned by the variables in $B$ (as in Section 2), and $\mathbf{q}$ is the $k$-vector space spanned by $\mathbf{m}$ and $y$.

Let $V_{d}$ be a $W_{d}$-monomial space; we have the disjoint union $\left\{V_{d}\right\}=\bigsqcup_{0 \leq j \leq d}$ $y^{d-j}\left\{U_{j}\right\}$ where each $U_{j}$ is a monomial space in $W / y$. Let $F_{j}$ be the lexification of the space $U_{j}$ in $W / y$. Consider the $W_{d}$-monomial space $T_{d}$ defined by $\left\{T_{d}\right\}=$ $\bigsqcup_{0 \leq j \leq d} z^{d-j}\left\{F_{j}\right\}$. Clearly, $\left|T_{d}\right|=\left|V_{d}\right|$. We call $T_{d}$ the $y$-compression of $V_{d}$.

Lemma 4.2. Let $V_{d}$ be a $W_{d}$-monomial space, and let $T_{d}$ be its y-compression. Then $\left|T_{d}\right|=\left|V_{d}\right|$ and $\left|\mathbf{q} T_{d}\right| \leq\left|\mathbf{q} V_{d}\right|$.

Proof. The proof is based on the same idea as the proof of Lemma 3.10. We write $\left\{V_{d}\right\}=\coprod_{0 \leq j \leq d} y^{d-j}\left\{U_{j}\right\}$ and $T_{d}=\coprod_{0 \leq j \leq d} y^{d-j}\left\{F_{j}\right\}$, where the $F_{j}$ are $B / M$-lex satisfying $\left|F_{j}\right|=\left|U_{j}\right|$. Then, as in the proof of Lemma 3.10, we have the disjoint unions

$$
\begin{aligned}
& \left\{\mathbf{q} V_{d}\right\}=\coprod_{0 \leq j \leq d} y^{d-i+1}\left\{U_{j}+\mathbf{m} U_{j-1}\right\} \\
& \left\{\mathbf{q} T_{d}\right\}=\coprod_{0 \leq j \leq d} y^{d-i+1}\left\{F_{i}+\mathbf{m} F_{j-1}\right\}
\end{aligned}
$$

and we have the inequalities

$$
\left|F_{i}+\mathbf{m} F_{j-1}\right|=\max \left\{\left|F_{j}\right|,\left|\mathbf{m} F_{j-1}\right|\right\} \leq \max \left\{\left|U_{j}\right|,\left|\mathbf{m} U_{j-1}\right|\right\} \leq\left|U_{j}+\mathbf{m} U_{j-1}\right|,
$$


where the middle inequality holds because $B / M$ is Macaulay-Lex. Since multiplication by $y$ is injective, we get

$$
\left|y^{d-i+1}\left(F_{i}+\mathbf{m} F_{j-1}\right)\right| \leq\left|y^{d-i+1}\left(U_{j}+\mathbf{m} U_{j-1}\right)\right| .
$$

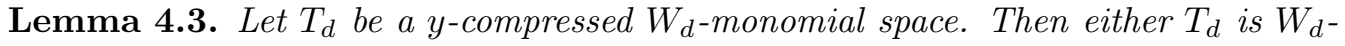
lex, or there exists a $W_{d}$-monomial space $F_{d}$, such that $F_{d}$ is strictly lexicographically greater than $T_{d},\left|F_{d}\right|=\left|T_{d}\right|$, and $\left|\mathbf{q} F_{d}\right| \leq\left|\mathbf{q} T_{d}\right|$; (here "lexicographically greater" means that we order the monomials in $\left\{F_{d}\right\}$ and $\left\{T_{d}\right\}$ lexicographically, and then compare the two ordered sets lexicographically).

Proof. Let $r$ be as large as possible among the numbers such that we can write

$$
T_{d}=y^{d-r} P \oplus\left(\bigoplus_{i>r} y^{d-i} L_{i}\right)
$$

with $P$ a lex segment of $W_{d}$. Such an $r$ always exists, as we can if necessary take $r=0$.

If $r=d$, then $T_{d}$ is $W_{d}$-lex and we are done. If not, then $y P+L_{r+1}$ is not lex in $W$. Let $m$ be the lex-greatest monomial of $W_{r+1}$ such that $m \notin y P+L_{r+1}$. We consider two cases depending on whether $y$ divides $m$ or not.

Suppose that $y$ divides $m$. Let $u$ be the lex-least monomial of $y P+L_{r+1}$. Since $P$ is lex and $y$ does not divide $m$, it follows that $y$ does not divide $u$. Let $Q$ be the $k$-vector space spanned by $\{Q\}$, defined by

$$
\{Q\}=\left(\{y P\} \cup\left\{L_{r+1}\right\} \cup\{m\}\right) \backslash\{u\} .
$$

Set

$$
F_{d}=y^{d-r-1} Q \oplus\left(\bigoplus_{i>r+1} y^{d-i} L_{i}\right)
$$

Now, $\left\{F_{d}\right\} \backslash y^{d-r-1} m=\left\{T_{d}\right\} \backslash y^{d-r-1} u$. Hence, $\left\{F_{d}\right\}$ is strictly lexicographically greater than $T_{d}$. We will compare $\left\{\mathbf{q} F_{d}\right\}$ and $\left\{\mathbf{q} T_{d}\right\}$. The set $\left\{\mathbf{m} y^{d-r-1} m\right\}$ is contained in $\left\{\mathbf{q} T_{d}\right\}$, so we have $\mathbf{q} F_{d} \backslash\left(\mathbf{q} F_{d} \cap \mathbf{q} T_{d}\right) \subseteq\left\{y^{d-r} m\right\}$. Furthermore, we will show that $y^{d-r} u \notin\left\{\mathbf{q} F_{d}\right\}$. Suppose the opposite. Hence, there exists a $q$ such that $y^{d-r} u=x_{q}\left(y^{d-r} \frac{u}{x_{q}}\right)$, where $\frac{u}{x_{q}} \in P$. But $y \frac{u}{x_{q}} \in y P$ is lex-smaller than $u$; this contradicts the choice of $u$. Hence $\left\{\mathbf{q} T_{d}\right\} \backslash\left(\mathbf{q} F_{d} \cap \mathbf{q} T_{d}\right) \supseteq\left\{y^{d-r} u\right\}$. Therefore, we have the desired inequality $\left|\mathbf{q} F_{d}\right| \leq\left|\mathbf{q} T_{d}\right|$. The lemma is proved in this case.

It remains to consider the case when $m$ is not divisible by $y$. In this case, $m$ is the lex-greatest monomial not divisible by $y$ that is lex-smaller than all the monomials in $\left\{L_{r+1}\right\}$. Set $z=x_{\max (m)}$. In our construction we will use the set

$$
N=\left\{u \in y P \mid u \prec_{\text {lex }} m \text { and }\left(\frac{z}{y}\right)^{e_{u}} u \neq 0 \text { in } B / M\right\},
$$

where $e_{u}$ is the largest power of $y$ dividing $u$. We will show that $N \neq \emptyset$ because $\frac{y}{z} m \in N$. Since $m$ is the lex-greatest monomial missing in $m \notin y P+L_{r+1}$, it follows that there exists a monomial $y m^{\prime} \in y P$ that is lex-smaller than $m$. Therefore, $m^{\prime}$ is 
(non-strictly) lex-smaller than $\frac{m}{z}$. As $m^{\prime} \in P$ and $P$ is lex, it follows that $\frac{m}{z} \in P$. Thus, $\frac{y}{z} m \in N$ as desired.

We will need three of the properties of $N$ :

\section{Claim.}

1. $m$ is (non-strictly) lex-greater than all the monomials in $\frac{z}{y} N$.

2. $\frac{z}{y} N \cap\left\{L_{r+1}\right\}=\emptyset$.

3. $\frac{z}{y} N \cap\{y P\} \subseteq N$.

We will prove the claim. (3) is clear. (2) follows from (1) and the fact that in the considered case $m$ is the lex-greatest monomial not divisible by $y$ that is lex-smaller than all the monomials in $\left\{L_{r+1}\right\}$. We will prove (1). Write

$$
m=x_{1}^{a_{1}} x_{2}^{a_{2}} \ldots z^{a_{z}} \quad \text { and } \quad u=x_{1}^{b_{1}} x_{2}^{b_{2}} \ldots z^{b_{z}} w y^{b_{y}},
$$

where $w$ is not divisible by $x_{1}, \ldots, z$ or by $y$. Suppose that the monomial $\frac{z}{y} u=$ $x_{1}^{b_{1}} x_{2}^{b_{2}} \ldots z^{b_{z}+1} w y^{b_{y}-1}$ is lex-greater than $m$. On the other hand, $m$ is lex-greater than $u$. It follows that $a_{j}=b_{j}$ for $j<\max (m)$ and $b_{z}<a_{z} \leq b_{z}+1$. Since the monomials have the same degree, it follows that $a_{z}=b_{z}+1, w=1$, and $b_{y}=1$. Hence $m=\frac{z}{y} u$. The claim is proved.

Let $Q$ be the $k$-vector space such that

$$
\{Q\}=\left(\left\{y P+L_{r+1}\right\} \backslash N\right) \cup \frac{z}{y} N .
$$

By the claim above, it follows that we have the disjoint union $\{Q\}=\left\{L_{r+1}\right\} \amalg y P \backslash$ $N \amalg \frac{z}{y} N$. Clearly, $|Q|=\left|L_{r+1} \oplus y P\right|$.

We consider the set

$$
F_{d}=y^{d-r-1} Q \oplus\left(\bigoplus_{i>r+1} y^{d-i} L_{i}\right)
$$

It is clear that $\left|F_{d}\right|=\left|T_{d}\right|$. Since $y^{d-r-1} m \in F_{d}$, we see that $F_{d}$ is strictly lexicographically greater than $T_{d}$. We will show that the inequality $\left|\mathbf{q} F_{d}\right| \leq\left|\mathbf{q} T_{d}\right|$ holds. Set $U=L_{r+1} \oplus y P$ and $V=\oplus_{i>r+1} y^{d-i} L_{i}$.

Since

$$
|\mathbf{q} Q|-|\mathbf{q} U|=-\mid\left\{t \in \mathbf{q} N \backslash\left(\mathbf{q} N \cap \mathbf{q}(U \backslash N) \mid \frac{z}{y} t=0\right\} \mid \leq 0\right.
$$

it follows that $|\mathbf{q} Q| \leq|\mathbf{q} U|$. Furthermore, we have

$$
\begin{aligned}
& \left|\mathbf{q} F_{d}\right| \\
& =\left|\mathbf{q} y^{d-r-1} Q\right|+|\mathbf{q} V|-\left|\mathbf{q} V \cap \mathbf{q} y^{d-r-1} Q\right| \\
& =\left|\mathbf{q} y^{d-r-1} Q\right|+|\mathbf{q} V|-\mid y^{d-r-1}\left(L_{r+2} \cap \mathbf{m}\{v \in Q \mid y \text { does not divide } v\}\right) \mid \\
& \leq\left|\mathbf{q} y^{d-r-1} U\right|+|\mathbf{q} V|-\mid y^{d-r-1}\left(L_{r+2} \cap \mathbf{m}\{v \in Q \mid y \text { does not divide } v\}\right) \mid \\
& \leq\left|\mathbf{q} y^{d-r-1} U\right|+|\mathbf{q} V|-\mid y^{d-r-1}\left(L_{r+2} \cap \mathbf{m}\{v \in U \mid y \text { does not divide } v\}\right) \mid \\
& =\left|\mathbf{q} T_{d}\right| ;
\end{aligned}
$$

the first inequality holds because multiplication by $y$ is injective, the second holds by set containment. 
We are ready for the proof of Theorem 4.1:

Proof. Let $V_{d}$ be a $W_{d}$-monomial space. If $V_{d}$ is not $W$-lex, apply Lemmas 4.2 and 4.3 to obtain a $y$-compressed $W_{d}$-monomial space $F_{d}$ which is strictly greater lexicographically than $V_{d}$ and satisfies $\left|F_{d}\right|=\left|V_{d}\right|$ and $\left|\mathbf{q} F_{d}\right| \leq\left|\mathbf{q} V_{d}\right|$. If $F_{d}$ is not $W$-lex, we can apply the lemmas again. After finitely many steps, the process must terminate in a lexicographic monomial space. Hence $W$ is $d$-pro-lex for all degrees $d \geq 0$, and so is Macaulay-Lex.

\section{Lexicographic quotients}

Theorem 5.1. If $M$ is Macaulay-Lex and $N$ is a B/M-lex ideal, then $M+N$ is Macaulay-Lex.

The theorem follows immediately from the following result:

Proposition 5.2. Fix a degree $d \geq 1$. If $M$ is $(d-1)$-pro-lex and $N$ is a $B / M$-lex ideal, then $M+N$ is $(d-1)$-pro-lex.

Proof. Throughout this proof, for a monomial space $\bar{V}$ in $B /(M+N)$, we denote by $V$ the $k$-vector space spanned by $\{\bar{V}\}$ in $B / M$.

Let $\bar{S}_{d-1}$ be a monomial space in $(B /(M+N))_{d-1}$. Let $\bar{L}_{d-1}$ be the $B /(M+N)$ lexification of $\bar{S}_{d-1}$. Set $\bar{L}_{d}$ to be the $k$-vector space spanned by $\mathbf{m}\left\{\bar{L}_{d-1}\right\}$ and $\bar{S}_{d}$ be the $k$-vector space spanned by $\mathbf{m}\left\{\bar{S}_{d-1}\right\}$. We will prove that

$$
\left|\bar{L}_{d}\right|^{B /(M+N)} \leq\left|\bar{S}_{d}\right|^{B /(M+N)} .
$$

First, we assume that the ideal $N$ has no minimal generators in degree $d$.

Note that $N_{d-1}+L_{d-1}$ is a $B / M$-lex-segment. Therefore, $N_{d-1}+L_{d-1}$ is the $B / M$-lexification of $N_{d-1}+S_{d-1}$ in the $\operatorname{ring} B / M$. Since $M$ is $(d-1)$-pro-lex, the following inequality holds:

$$
\left|N_{d}+L_{d}\right|^{B / M} \leq\left|N_{d}+S_{d}\right|^{B / M} .
$$

On the other hand,

$$
\begin{aligned}
& \left|N_{d}+L_{d}\right|^{B / M}=\left|N_{d}\right|^{B / M}+\left|L_{d}\right|^{B / M}-\left|N_{d} \cap L_{d}\right|^{B / M} \\
& \left|N_{d}+S_{d}\right|^{B / M}=\left|N_{d}\right|^{B / M}+\left|S_{d}\right|^{B / M}-\left|N_{d} \cap S_{d}\right|^{B / M}
\end{aligned}
$$

Therefore, we obtain the inequality

$$
\left|L_{d}\right|^{B / M}-\left|N_{d} \cap L_{d}\right|^{B / M} \leq\left|S_{d}\right|^{B / M}-\left|N_{d} \cap S_{d}\right|^{B / M} .
$$

Note that the left hand-side is equal to $\left|\bar{L}_{d}\right|^{B /(M+N)}$ whereas the right-hand side is equal to $\left|\bar{S}_{d}\right|^{B /(M+N)}$. Thus, we get the desired inequality

$$
\left|\bar{L}_{d}\right|^{B /(M+N)} \leq\left|\bar{S}_{d}\right|^{B /(M+N)} .
$$

Now, suppose that $N$ has minimal monomial generators in degree $d$.

If $L_{d} \subseteq N_{d}$, then

$$
0=\left|\bar{L}_{d}\right|^{B /(M+N)} \leq\left|\bar{S}_{d}\right|^{B /(M+N)} .
$$


Suppose that $L_{d} \not \subset N_{d}$. Set $Q=\left\{N_{d}\right\} \backslash\left\{\mathbf{m} N_{d-1}\right\}$. Since both $\mathbf{m} N_{d-1}+L_{d}$ and $N_{d}$ are $B / M$-lex-segments, it follows that one of them contains the other. Hence $\left\{L_{d}\right\} \supseteq Q$, so

$$
\left|\bar{L}_{d}\right|^{B /(M+N)}=\left|L_{d}\right|^{B /\left(M+\left(N_{d-1}\right)\right)}-|Q| .
$$

The argument above (for the case when the ideal is $(d-1)$-generated) can be applied to $N_{d-1}$, and it yields

$$
\left|L_{d}\right|^{B /\left(M+\left(N_{d-1}\right)\right)} \leq\left|S_{d}\right|^{B /\left(M+\left(N_{d-1}\right)\right)} .
$$

Therefore we have

$$
\begin{aligned}
\left|\bar{L}_{d}\right|^{B /(M+N)}=\left|L_{d}\right|^{B /\left(M+\left(N_{d-1}\right)\right)}-|Q| & \\
\leq\left|S_{d}\right|^{B /\left(M+\left(N_{d-1}\right)\right)}-|Q| & \leq\left|S_{d}\right|^{B /\left(M+\left(N_{d-1}\right)\right)}-\left|Q \cap\left\{S_{d}\right\}\right| \\
& =\left|\bar{S}_{d}\right|^{B /(M+N)} .
\end{aligned}
$$

Macaulay's Theorem [Ma] says that 0 is pro-lex. Hence, Theorem 5.1 applied to $M=0$ yields the following:

Corollary 5.3. If $U$ is a B-lex ideal then it is Macaulay-Lex.

Remark 5.4. Following [Sh], we say that a monomial ideal $M$ in $B$ is piecewise lex if, whenever $\mathbf{x}^{\mathbf{a}} \in M, \mathbf{x}^{\mathbf{b}} \succ_{\text {lex }} \mathbf{x}^{\mathbf{a}}$, and $\max \left(\mathbf{x}^{\mathbf{b}}\right) \leq \max \left(\mathbf{x}^{\mathbf{a}}\right)$, we have $\mathbf{x}^{\mathbf{b}} \in M$. Shakin [Sh] proved that if $M$ is a piecewise lex ideal in B, then it is Macaulay-Lex. This result can be proved differently using our technique as follows:

Proof. We induct on $n$. Let $\mathbf{x}^{\mathbf{a}_{1}}, \ldots, \mathbf{x}^{\mathbf{a}_{r}}$ be the minimal monomial generators of $M$ divisible by $x_{n}$. So the lex segment $L_{j}$ ending in $\mathbf{x}^{\mathbf{a}_{j}}$ must be contained in $M$. Set $N=M \cap k\left[x_{1}, \cdots, x_{n-1}\right]$. Then $N$ is piecewise lex and so by induction is MacaulayLex in $k\left[x_{1}, \cdots, x_{n-1}\right]$. By Theorem $4.1, N$ is Macaulay-Lex in $B$. By induction on $j$, we conclude that $\left(N+L_{1}+\cdots+L_{j-1}\right)+L_{j}$ is Macaulay-Lex by Theorem 5.1 . Hence, $M=N+L_{1}+\cdots+L_{r}$ is Macaulay-Lex as well.

\section{Acknowledgements}

We thank Christopher Francisco, Mike Stillman, and Steven Sinnott for helpful discussions. Irena Peeva is partially supported by NSF.

\section{References}

[Bi] A. Bigatti, Upper bounds for the Betti numbers of a given Hilbert function, Comm. in Algebra 21 (1993) 2317-2334.

[CL] G. Clements and B. Lindström, A generalization of a combinatorial theorem of Macaulay, J. Combinatorial Theory 7 (1969) 230-238.

[EK] S. Eliahou and M. Kervaire, Minimal resolutions of some monomial ideals, J. Algebra 129 (1990) 1-25.

[Gr] M. Green, Generic initial ideals, in Six lectures on commutative algebra, Birkhäuser, Progress in Mathematics 166 (1998) 119-185.

[Ha] R. Hartshorne, Connectedness of the Hilbert scheme, Inst. Hautes Études Sci. Publ. Math. 29 (1966) 5-48.

[Hu] H. Hulett, Maximum Betti numbers of homogeneous ideals with a given Hilbert function, Comm. Algebra 21 (1993), 2335-2350. 
[Ka] G. Katona, A theorem for finite sets, Theory of Graphs (P. Erdös and G. Katona, eds.), Academic Press, New York (1968) 187-207.

[Kr] J. Kruskal, The number of simplices in a complex, Mathematical Optimization Techniques (R. Bellman, ed.), University of California Press, Berkeley/Los Angeles (1963) 251-278.

[Ma] F. Macaulay, Some properties of enumeration in the theory of modular systems, Proc. London Math. Soc. 26 (1927) 531-555.

[Pa] K. Pardue, Deformation classes of graded modules and maximal Betti numbers, Illinois J. Math. 40 (1996), 564-585.

[Sh] D. A. Shakin, Piecewise lexsegment ideals, Mat. Sb. 194 (2003) 1701-1724.

Department of Mathematics, Cornell University, IthacA, NY 14853 